\title{
ORBITS OF HIGHEST DIMENSION
}

\author{
BY \\ D. MONTGOMERY AND C. T. YANG
}

1. Introduction. Let $G$ be a compact connected Lie group which acts on an $n$-dimensional manifold $X$. An earlier paper [3] has discussed the exceptional orbits of highest dimension but for the main result it was required that $X$ be differentiable and that $G$ act differentiably. In the present paper we extend the result to the nondifferentiable case and include some additional related material. For this extension $X$ is taken to be a certain kind of generalized manifold recently studied by Yang [8] and results of this study are used throughout this paper.

Let $r$ be the highest dimension of any orbit of $G$ in its action on $X$ and let $F$ be the set of singular points, that is, points on orbits of dimension less than $r$. It is known $[2 ; 8]$ that $F$ is a closed set of dimension $\leqq n-2$. The set of points on exceptional orbits of dimension $r$ is denoted by $E$; at a point of $E$ the isotropic group is discontinuous and, to speak intuitively, an orbit in $E$ is characterized by the fact that arbitrarily near it there are orbits which wind around it more than once. The result shown before and here extended is that if the $(n-1)$-homology of $X$ is trivial (for example $N=E^{n}$ or $S^{n}$ ) then

$$
\operatorname{dim}(E \cup F) \leqq n-2 .
$$

Homology is always Cech homology and is either for $X$ if $X$ is compact, or of the one point compactification if $X$ is not compact. Either way we use the symbols $H_{k}(X), H_{k}(X, A)$, etc.

2. Definitions and preliminaries. Let $X$ be a $(\mathfrak{B}, n)$-manifold [8], that is a generalized manifold using for coefficients the group $\mathbb{B}$ which is group of the reals mod one. Let $G$ be a compact connected Lie group acting on $X$ with $r$ being the highest dimension of any orbit. As in [8] we let

$$
\begin{aligned}
G_{x} & =\text { isotropic subgroup of } G \text { at } x, \\
G_{x}^{*} & =\text { identity component of } G_{x}, \\
m(x) & =\operatorname{order} G_{x} / G_{x}^{*}, \\
X_{u, v} & =\{x: x \in X, \operatorname{dim} G(x)=u, m(x)=v\}, \\
F & =\bigcup\left\{X_{u v}: u<r\right\} .
\end{aligned}
$$

As already mentioned $[2 ; 8]$

$$
\operatorname{dim} F \leqq n-2 .
$$

Received by the editors July 2, 1956. 
Let

$$
s=\inf \{m(x): x \in X-F, \operatorname{dim} G(x)=r\}, \quad E=U\left\{X_{r v}: v>s\right\} .
$$

The set $E \cup F$ is closed and as stated above it will be shown under certain conditions to have dimension $\leqq n-2$; therefore under these conditions $X-(E \cup F)$ is a fibre bundle with orbits as fibres.

Let $p$ be a point of $X-F$, and let $K$ be a connected slice at $p[5 ; 6]$ as modified in [8]. Let $G_{K}$ be the subgroup of $G_{p}$ which leaves all of $K$ fixed; $G_{K}$ is a normal subgroup of $G_{p}$ containing $G_{p}^{*}$. Therefore

$$
H=G_{p} / G_{K}
$$

is a finite group acting effectively on $K$.

Lemma 1. If $p \in\left(X_{r, s}\right)-\cap E$, then for every $x \in K, s$ is a factor of $m(x)$ and $m(x) / s$ is equal to the order of $H_{x}$. Therefore a point $x$ of $K$ belongs to $E$ if and only if $H_{x}$ is not trivial.

Proof. Since $p \in\left(X_{r, s}\right)^{-}, K \cap X_{r, s}$ is not null and there is a point $y \in K \cap X_{r, 8}$. Then

$$
G_{K} \subset G_{y}
$$

Suppose $G_{K} \neq G_{y}$. Then there is an element $g$ of $G_{y}$ not contained in $G_{K}$. Then $g G_{K}$, as an element of $H$, is not the identity and a certain power of $g G_{K}$, denoted by $T$, is of prime order. Since $K$ is a $(\mathfrak{B}, n-r)$ manifold it follows [8, Lemma 15] that the fixed point set of $T$ is nowhere dense in $K$. But $G_{x}$ is continuous at $y$ and therefore is constant over a neighborhood of $y$ in $K$. This means that $y$ is an inner point of the fixed point set of $g G_{K}$ and of $T$. This is a contradiction and proves that $G_{K}=G_{y}$ and the assertions of the lemma now follow. This completes the proof.

Lemma 2. $\operatorname{dim} E \leqq n-1$ and $\left(X_{r, s}\right)^{-}=X$.

Proof. Let $p$ be a point of $E$ and let $K$ and $H$ be as above. Suppose that $\left(X_{r, s}\right)^{-} \neq X$. Then $\left(X_{r, s}\right)^{-}-$Int $\left(X_{r, s}\right)^{-}$is not null and is $(n-1)$-dimensional $[8$, Lemma 14] so that it cannot be contained in $F$. Hence we may choose a point of $\left(X_{r, s}\right)^{-}-\operatorname{Int}\left(X_{r, s}\right)^{-}$as $p$. Then $K-\left(X_{r, s}\right)^{-}$is non-null and open in $K$ and is contained in $E$. Therefore

$$
K \cap E=K-X_{r, s}
$$

is somewhere dense in $K$.

By Lemma 1, a point of $K$ belongs to $E$ if and only if it is a fixed point of some nontrivial subgroup of $H$. By [8, Theorem 1], the fixed point set of a nontrivial subgroup of $H$ is nowhere dense in $K$. It follows that $K \cap E$ is nowhere dense in $E$. Hence we have obtained a contradiction which proves that $\left(X_{r, s}\right)^{-}=X$. 
Since $E \cup F=\left(X_{r, s}\right)^{-}-X_{r, s}, E \cup F$ is nowhere dense. It follows from [8, Lemma 14] that $\operatorname{dim}(E \cup F) \leqq n-1$ and therefore $\operatorname{dim} E \leqq n-1$ which completes the proof.

For any integer $k>1$ let

$$
E_{k}=\{x: x \in E, k s \mid m(x)\}
$$

where $k s \mid m(x)$ means that $k s$ is a factor of $m(x)$.

Lemma 3. $E=U\left\{E_{k}: k\right.$ prime $\}$ and the set

$$
E^{\prime}=\bigcup\left\{E_{k}: k \text { an odd prime or } 4\right\}
$$

is of dimension $\leqq n-2$.

Proof. Let $p$ be a point of $E$ and $K$ a slice at $p$ as above. It follows from $\left(X_{r, s}\right)^{-}=X$, that $K \cap X_{r, s}$ is non-null. If $y$ is a point of $K \cap X_{r, s}$, then

(a) $G_{y} \subset G_{x}$,

(b) $m(p)$ is the order of $G_{p} / G_{p}^{*}$,

(c) $s$ is the order of $G_{y} / G_{p}^{*}$.

Therefore $s$ is a factor of $m(p)$. Since $p \in E, s \neq m(p)$, and for some prime number $k, k s$ is a factor of $m(p)$ so $p \in E_{k}$. Hence

$$
E=U\left\{E_{k} ; k \text { prime }\right\} \text {. }
$$

Suppose that $E^{\prime}$ is of dimension $>n-2$. It follows from Lemma 2 that the dimension of $E^{\prime}$ is $n-1$.

By [8, Lemma 7], there is some $k>2$ such that $X_{r, k s}$ is $(n-1)$-dimensional. Therefore there is a point $p$ of $X_{r, k s}$ such that every neighborhood of $p$ in $X_{r, k s}$ is $(n-1)$-dimensional [ 8 , Corollary to Lemma 4]. Let $K$ be a connected slice at $p$ and let $Q$ be an open $r$-cell in $G$ which contains the identity and is such that $Q(K)$ is a neighborhood of $p$ and that $(g, x) \rightarrow g(x)$ defines a homeomorphism of $Q \times K$ onto $Q(K)$.

Let $G_{K}$ be the subgroup of $G_{p}$ which leaves every point of $K$ fixed and let $H=G_{p} / G_{K}$. It follows from Lemma 1 that $H$ is a finite group of order $k$. Since $k>2$ and $K$ is a $(\mathfrak{B}, n-r)$ manifold, it follows from [8, Theorem 1] that $K \cap X_{r, k s}$, the fixed point set of $H$ is of dimension $\leqq n-r-2$. Hence by [8, Lemma 5] the dimension of $X_{r, k s} \cap Q(K)$ is of dimension $\leqq n-2$, contrary to the result above. This contradiction completes the proof.

Let $X^{*}$ be the orbit space of $X$. Whenever $S$ is a subset of $X$, the image of $S$ is $X^{*}$ is denoted by $S^{*}$.

Lemma 4. The set $X_{r, s}^{*}$ is connected and hence $X_{r, s}$ is a fibre bundle with orbits as fibres. If $G$ is connected then $X_{r, s}$ is connected.

Proof. Let $M$ be a subset of $X_{r, s}$ such that $G(M)=M$ and $M^{*}$ is a component of $X_{r, s}^{*}$. Suppose that $M \neq X_{r, s}$. Then

$$
\bar{M}-\text { Int } \bar{M} \neq \varnothing \text {. }
$$


Since $\bar{M}-$ Int $\bar{M}$ is an $(n-1)$-dimensional subset of $E \cup F$ and since $E^{\prime} \cup F$ is $(n-2)$-dimensional, it follows that there is a point $p$ of $\bar{M}-\operatorname{Int} \bar{M}$ belonging to $E-E^{\prime}=X_{r, 2 s}$.

Let $K, H$ be as before. It follows from Lemma 1 that $H$ is of order 2. Denote by $T$ the nonzero element of $H$. Then there exists by [8, Lemma 16] a neighborhood $A$ of $p$ in $K$ such that $A-E$ has exactly two components $C$ and $T(C)$. Since $p \in \bar{M}$, we may assume $C \subset M$. Therefore

$$
M=G(M) \supset G(C) \supset T(C)
$$

and hence $p \in \operatorname{Int} \bar{M}$ contrary to assumption. This proves the lemma.

\section{Existence of a nontrivial relative cycle in $\bar{E}$.}

Lemma 5. If $\operatorname{dim} E=n-1$ then there is a nonzero element $\boldsymbol{z}^{\prime}$ of

$$
H_{n-1}\left(\bar{E}, \bar{E}-E ; \mathfrak{\Im}_{2}\right) \text {. }
$$

Let $p$ be a point of $E$ and let $K, H$ be as above. Let $Q$ be a small open cell in $G$ which is a local cross-section of the cosets of $G_{p}$ in $G$ at the identity and such that

$$
\left(Q \cup Q^{-1}\right)^{2} G_{p}^{*}\left(Q \cup Q^{-1}\right)^{2} \cap G_{p}=G_{p}^{*},
$$

and $(g, x) \rightarrow g(x)$ is a homeomorphism of $Q \times K$ onto $Q(K)$.

Let $U$ be a neighborhood of $p$ satisfying the following properties:

(i) $\bar{U} \subset Q(K)$.

(ii) If $T$ is an element of $H$ of order 2 and $L_{T}$ is the fixed point set in $T$ in $K$, then $L_{T} \cap U$ is contained in a component of $L_{T}$.

By hypothesis $\operatorname{dim} E=n-1$ so $\operatorname{dim} K \cap E=n-r-1$. We consider elements $T$ such that $\operatorname{dim} U \cap L_{T}=n-r-1$, and we shall show that for any such $T, L_{T}$ has the property $P_{n-r-1}\left(\mathbb{E}_{2}\right)$ at some point of $U \cap L_{T}$ and hence at all such points. By [8, Lemma 14] there is a point $x \in L_{T} \cap U$ and a neighborhood $Q$ of $x$ in $K \cap U$ such that every neighborhood of $x$ in $K \cap U$ meets at least two components of $Q-\left(L_{T} \cap U\right)$. There is a fundamental $\left(\mathfrak{S}_{2}, n-r\right)$-pair $\left(W,\left[\mathfrak{G}_{2}\right]\right)$ with $W$ connected and contained in $Q$, and $W-\left(L_{T} \cap U\right)$ contains at least two components. Denote one of these components by $A$. Let (as in the proof of $[8$, Lemma 14])

$$
M=[(\bar{A}-A) \cap W]^{-}, \quad N=M-W .
$$

Then $M, N$ are compact subsets of $L_{T} \cap U$ and the natural homomorphism of $H_{n-r-1}\left(M, N ; \mathfrak{S}_{2}\right)$ into $H_{n-r-1}\left(\bar{A}-A, \bar{A}-W ; \mathfrak{S}_{2}\right)$ is one-one and onto. Since $H_{n-r}\left(\bar{A}, \bar{A}-W, \mathfrak{S}_{2}\right)=0$ and $H_{n-r}\left(\bar{A}, \bar{A}-A ; \mathfrak{夭}_{2}\right)$ is isomorphic to $\mathfrak{夭}_{2}$ it follows from

$$
\begin{aligned}
H_{n-r-1}\left(M, N ; \mathfrak{\Xi}_{2}\right) & \stackrel{\approx}{\rightleftarrows} H_{n-r-1}\left(\bar{A}-A, \bar{A}-U: \mathfrak{\Xi}_{2}\right) \stackrel{\partial}{\longleftarrow} H_{n-r}\left(\bar{A}, \bar{A}-A ;\left(\mathfrak{\Xi}_{2}\right)\right. \\
& \longleftarrow H_{n-r}\left(\bar{A}, \bar{A}-U, \mathfrak{\Xi}_{2}\right)
\end{aligned}
$$


that $H_{n-r-1}\left(M, N, \mathfrak{\mho}_{2}\right) \neq 0$.

It follows that at a point $p$ of $M-N, L_{T}$ has the property $P_{n-r-1}\left(\mathfrak{S}_{2}\right)$.

It follows from [8, Lemmas 11,12 , and Remark 3$]$ that there is a fundamental $\left(\mathfrak{C}_{2}, n-r-1\right)$-pair $\left(L_{T} \cap U, S^{\prime}\right)$. Then there is a fundamental $\left(\mathfrak{C}_{2}\right.$, $n-1)$-pair $\left(Q\left(L_{T}\right) \cap U, S\right)$. Now let

$$
B=\{x: x \in E, \operatorname{dim} E \text { at } x \text { is } n-1\} .
$$

For any $p$ in $B$, there is a slice at $p$ and a corresponding cell $Q$ and for some $T, p \in Q\left(L_{T}\right)$ and $Q\left(L_{T}\right)$ has the property $P_{n-1}\left(\mathbb{E}_{2}\right)$. Observe that $B$ is the union of these sets of the form $Q\left(L_{T}\right)$ and that $\bar{B}-B C \bar{E}-E$.

As in [3] we associate with $\bar{B}$ a new space $R$ in the following manner. The points of $R$ are of the following kinds:

(1) points of $\bar{B}-B$,

(2) the points $p$ of $B$ which are "essentially" on only one set $Q\left(L_{T}\right)$; that is for such a $p$ there is only one $T$ leaving $p$ fixed so that the $Q\left(L_{T}\right)$ including $p$ is unique in the sense that any two such coincide in a neighborhood of $p$,

(3) the points $p$ of $B$ which are "essentially" on more than one $Q\left(L_{T}\right)$, (the number is always finite). For such a point $p$ there are a finite number of sets of the form $Q\left(L_{T}\right)$, call them $Y_{1}, \cdots, Y_{k}$ such that

$$
p \in Y_{i}, \quad i=1, \cdots, k
$$

and if $p$ is in any set of the form $Q\left(L_{T}\right)$ this set coincides with some $Y_{i}$ in a neighborhood of $p$. In this case $p$ is replaced by $k$ points, $p_{1}, \cdots, p_{k}$. The set $R$ is topologized by the following neighborhoods:

(a) A point of $\bar{B}-B$ has the same neighborhoods as before except that if the original neighborhood included a point $p$ of type (3) above, then the new neighborhood is to include $p_{1}, \cdots, p_{k}$.

(b) For a point $p$ of type (2) we choose the same neighborhoods as before which are connected and small enough to have property $P_{n-1}\left(\mathfrak{S}_{2}\right)$ throughout.

(c) For a point $p$ of type (3) we choose as a neighborhood of $p_{i}$ any open set in $Y_{i}$ which includes $p_{i}$, is connected, and has the property $P_{n-1}\left(\Im_{2}\right)$ throughout.

In $R$ the points of type (2) and (3) constitute a set $S$ at each point of which the property $P_{n-1}\left(\mathcal{E}_{2}\right)$ is valid. There is a natural map $f$ which satisfies

$$
f: R \rightarrow \bar{B}, \quad f: S \rightarrow B
$$

and the space $R$ is compact (at least if $X$ has been compactified).

In $S$ choose a component $S_{1}$. Then $\bar{S}_{1}$ carries a relative $(n-1)$-cycle mod 2 which does not bound. In order to see this we refer to [8, Lemma 12] where it has been shown that if $U$ is any bounded subset of $S_{1}$ then $I I_{n-1}\left(S_{1}, S_{1}-U, \mathfrak{C}_{2}\right)$ 
$\approx \mathfrak{G}_{2}$. By representing $\left(\bar{S}_{1}, \bar{S}_{1}-S_{1}\right)$ as an inverse limit we get the result stated above.

It follows that $f\left(\bar{S}_{1}\right)$ carries a relative $(n-1)$-cycle mod 2 which does not bound in $\bar{B}$, nor in $\bar{E}$.

This completes the proof.

4. Existence of an $(n-1)$-cycle. The following theorem applies to a space $X$ whether or not it is a manifold.

We prove the following theorem for $X$ compact although, as usual, it will apply to the locally compact case by our standard device of adding a point at infinity.

TheOREM 1. Let $G$ be a compact Lie group acting on a compact Hausdorff space $X$ with a singular set $F$. Let $B$ be an invariant open subset of $X-F$ satisfying

(a) $\mathrm{Bd} B C F(\mathrm{Bd} B=\bar{B}-B)$,

(b) $\operatorname{dim} \bar{B}=n-1, \operatorname{dim} \mathrm{Bd} B \leqq n-2$.

Then the natural homomorphism of $H_{n-1}\left(\bar{B} ; \mathfrak{V}_{2}\right)$ into $H_{n-1}\left(\bar{B}, \mathrm{Bd} B ; \mathfrak{C}_{2}\right)$ is onto.

For the proof suppose the theorem false. Then the boundary operator

$$
\text { ว: } H_{n-1}\left(\bar{B}, \mathrm{Bd} B ; \mathfrak{C}_{2}\right) \rightarrow H_{n-2}\left(\mathrm{Bd} B ; \mathfrak{C}_{2}\right)
$$

is not trivial. Therefore for some $z \in H_{n-1}\left(\bar{B}, \mathrm{Bd} B ; \mathcal{E}_{2}\right)$,

$$
\partial z \neq 0 \text {. }
$$

Let $Y$ be a minimal compact subset of $\mathrm{Bd} B$ such that

$$
(\partial z) \mid(\operatorname{Bd} B, Y)=0 .
$$

We wish now to prove the following: if $x \in Y$ and $V$ is any open set including $x$, then

$$
(\partial z) \mid(\operatorname{Bd} B, \operatorname{Bd} B-V) \neq 0 .
$$

Of course $Y$ is also minimal with respect to the following property: the map $H_{n-2}\left(Y ; \mathfrak{V}_{2}\right) \rightarrow H_{n-2}\left(\mathrm{Bd} B ; \mathfrak{V}_{2}\right)$ takes some homology class into $\partial z$.

There is a $w_{1} \in H_{n-2}\left(Y ; \quad \mathbb{E}_{2}\right)$ such that

$$
w_{1} \mid \mathrm{Bd} B=\partial z
$$

and assuming the statement above is false there is a $w_{2} \in H_{n-2}\left(\mathrm{Bd} B-V ; \mathfrak{G}_{2}\right)$ such that

$$
w_{2} \mid \operatorname{Bd} B=\partial z .
$$

Applying the Mayer-Vietoris sequence $[1$, p. 39] to $(Y \cup(\operatorname{Bd} B-V) ; Y$, 
$\mathrm{Bd} B-V)$ and recalling that $Y \cap(\mathrm{Bd} B-V)=Y-V$, we get

$$
\begin{aligned}
H_{n-2}\left(Y \cup(\mathrm{Bd} B-V) ; \mathfrak{夭}_{2}\right) & \stackrel{\phi}{\leftarrow} H_{n-2}\left(Y ; \mathfrak{\mho}_{2}\right)+H_{n-2}\left(\mathrm{Bd} B-V ; \mathfrak{夭}_{2}\right) \\
& \stackrel{\leftarrow}{\leftarrow} H_{n-2}\left(Y-V ; \mathfrak{夭}_{2}\right) .
\end{aligned}
$$

Since $Y$ is minimal the element $\left(w_{1}, w_{2}\right)$ is not covered by $\psi$ so it is sent by $\phi$ to a nonzero element of $H_{n-2}\left(Y \cup(\mathrm{Bd} B-V) ; \widehat{夭}_{2}\right)$ which however bounds in $\operatorname{Bd} B$ by $\left({ }^{*}\right)$ and $\left(^{* *}\right)$. This shows $[8$, Lemma 4$]$ that $\operatorname{dim} \operatorname{Bd} B \geqq n-1$ which contradicts the hypothesis. This contradiction completes the proof of the desired fact stated above.

Let $C$ be the totality of points $x$ of $\mathrm{Bd} B$ such that whenever $V$ is an open set including $x$,

$$
(\partial z) \mid(\mathrm{Bd} B, \mathrm{Bd} B-V) \neq 0 .
$$

Then $C$ is closed and as shown above is nonvacuous. Therefore there is a point $p$ of $C$ and an open neighborhood $U$ of $p$ such that whenever $x \in C \cap U$, $G_{x}$ is conjugate to $G_{p}$.

Let $K$ be a slice at $p$ and let $Q$ be an open cell in $G$ containing the identity and such that $Q(K)$ is an open neighborhood of $p$ contained in $U$ and $(g, x)$ $\rightarrow g(x)$ is a homeomorphism of $Q \times K$ onto $Q(K)$. Let $H \subset K$ be an open neighborhood of $p$ in $K$ (make the choice so $Q(H)$ is open) and let $k$ be the dimension of $G(p)$. Then $Q$ is $k$-dimensional and therefore we have a diagram as follows (we omit $\mathfrak{C}_{2}$ as understood for the remainder of the proof):

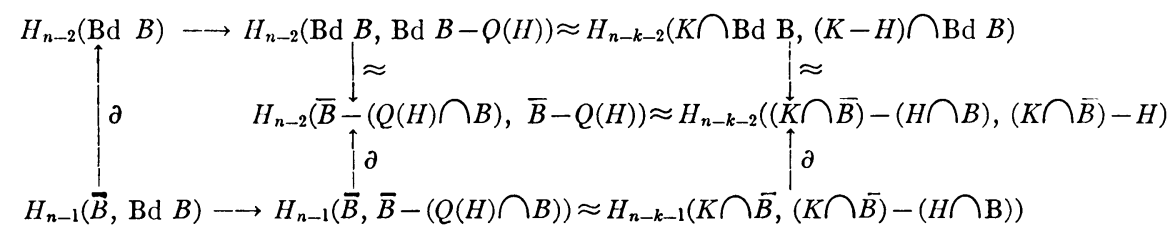

The isomorphisms from the second column to the third come from the fact that the spaces in the second column are obtained from those in the third column by multiplying by a $k$-cell [see 8 ]; the groups involved are taken modulo the appropriate boundaries.

Let $z^{\prime}$ be the image of $z$ in $H_{n-k-1}(K \cap \bar{B},(K \cap \bar{B})-(H \cap B))$ and let $v$ be the image of $\partial z$ in $H_{n-k-2}(K \cap \mathrm{Bd} B ;(K-H) \cap \mathrm{Bd} B)$. Then $z^{\prime}$ and $v$ have the same image, that is

$$
\partial z^{\prime}=v \mid((K \cap \bar{B})-(H \cap B),(K \cap \bar{B})-H) .
$$

Let $Y$ (not the same $Y$ as above) be a minimal compact subset of $K \cap B d B$ such that for some $v^{\prime} \in H_{n-k-2}(Y, Y \cap(K-H))$,

$$
v^{\prime} \mid(K \cap \mathrm{Bd} B,(K-H) \cap \mathrm{Bd} B)=v .
$$


It will now be shown that $Y \subset C$. Let $x$ be a point of $Y \cap H$ and let $W$ be an open neighborhood of $x$. There is an open neighborhood $H^{\prime}$ of $x$ in $K$, and an open neighborhood $Q^{\prime}$ of the identity in $Q$ such that $H^{\prime} \subset H, Q^{\prime}$ is an open $k$-cell, and $Q^{\prime}\left(H^{\prime}\right) \subset W$. By the minimality of $Y$

$$
v^{\prime} \mid\left(Y, Y \cap\left(K-H^{\prime}\right)\right) \neq 0 .
$$

This can be seen $[1$, p. 25] from the homology sequence of the triple $\left(Y, Y \cap\left(K-H^{\prime}\right), Y \cap(K-H)\right)$. Since $K \cap \mathrm{Bd} B$ is $(n-k-2)$-dimensional,

$$
v^{\prime} \mid\left(K \cap \operatorname{Bd} B,\left(K-H^{\prime}\right) \cap \operatorname{Bd} B\right) \neq 0 .
$$

By properties of the homology of a topological product of a space and a cell it follows that

$$
(\partial z) \mid\left(\operatorname{Bd} B, \operatorname{Bd} B-Q^{\prime}\left(H^{\prime}\right)\right) \neq 0 \text {. }
$$

Hence

$$
(\partial z) \mid(\mathrm{Bd} B, \mathrm{Bd} B-W) \neq 0 .
$$

Since $W$ is an arbitrary open neighborhood of $x$, it follows that $x \in C$. Hence $Y \cap H \subset C$ and since $Y \subset$ closure $(Y \cap H)$, it follows that $Y \subset C$.

The group $G_{p}$ is a transformation group of $K$. Let $f$ be the natural map of $K$ into its orbit space. Then we have the following diagram:

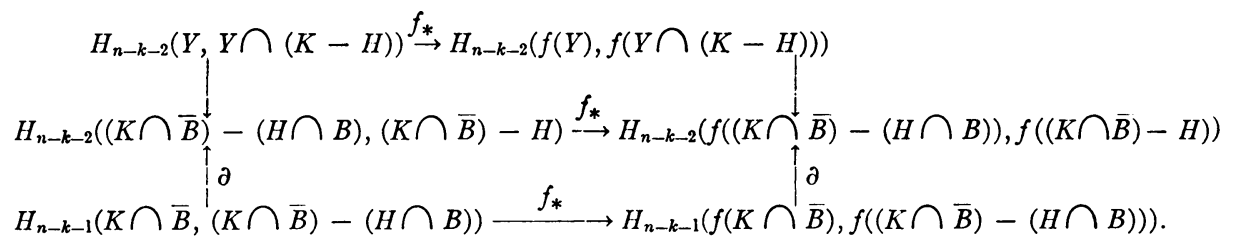

Since $Y \subset C \cap U, f$ is a homeomorphism on $Y$ and then $f_{*}\left(v^{\prime}\right) \neq 0$. Therefore $\partial f_{*}\left(z^{\prime}\right) \neq 0$ as $f((K \cap \bar{B})-(H \cap B))$ is $(n-k-2)$-dimensional. But $f(K \cap \bar{B})$ is $(n-k-2)$-dimensional $\left(\left[8\right.\right.$, Theorem 2]) and then $f_{*}\left(z^{\prime}\right)=0$. Hence we have arrived at a contradiction which proves the theorem.

For the following theorem we return to the assumption made at the beginning that $X$ is a manifold. If $X$ is not compact we compactify it by adding a point.

THEOREM 2. If $G$ is a compact Lie group acting on a ( $\mathfrak{B}, n)$-manifold $X$ and if $\operatorname{dim} E=n-1$ then there exists $z \neq 0$,

$$
z \in H_{n-1}\left(\bar{E} ; \mathfrak{\mho}_{2}\right) .
$$

Proof. This is a corollary of the preceding theorem and Lemma 5.

5. Statement and proof of main results. 
ThEOREM 3. Let $G$ be a compact connected Lie group acting on a (P, n)manifold $X$ where $H_{n-1}\left(X ; \mathfrak{E}_{2}\right)=0$. Then

$$
\operatorname{dim} E<n-1 \text {. }
$$

Proof. Assume the theorem is false. Then there exists $z, z \neq 0$,

$$
z \in H_{n-1}\left(\bar{E} ; \mathfrak{S}_{2}\right) .
$$

Our assumptions on $X$ and results on $\bar{E}$ give us the following exact sequence (the coefficient group $\mathfrak{C}_{2}$ is understood and omitted):

$$
0 \leftarrow H_{n-1}(\bar{E}) \leftarrow H_{n}(X, \bar{E}) \leftarrow H_{n}(X) \leftarrow 0 .
$$

By [8, Remark 7 and Corollary to Lemma 12] $H_{n}(X)$ is cyclic of order 2 and we have just mentioned that $H_{n-1}(\bar{E})$ is not trivial. Hence $H_{n}(X, \bar{E})$ contains more than two elements and therefore $X-\bar{E}$ is not connected because if it were, $H_{n}(X, \bar{E})$ would be cyclic of order $2[8$, loc. cit.].

Now let $W$ be one of the components of $X-\bar{E}$. Since $G$ is connected,

$$
G(W)=W .
$$

Now select a point $p$ in

$$
E \cap(\bar{W}-\operatorname{Int} \bar{W})
$$

where $G_{x}$, taken on

$$
E \cap(\bar{W}-\text { Int } \bar{W}),
$$

is continuous, and take a slice $K$ at $p$. Then $G_{p} / G_{K}$ is cyclic of order 2 and by [8, Lemma 16], $E \cap(\bar{W}-\operatorname{Int} \bar{W})$ divides $K$, locally into two domains which are interchanged by the nontrivial element of $G_{p} / G_{K}$. Hence both these domains are in $W$, since, clearly at least one of them contains a point of $W$ (and so is in $W$ ) and $W$ is invariant under $G$. But then $p$ is not in $\bar{W}-$ Int $\bar{W}$. This contradiction proves the theorem.

THEOREM 4. Let $G$ be a compact connected Lie group acting on a (P, n)manifold and let $x$ be fixed for every $g \in G$. Then there is an open set $V$ including $x$ such that

$$
\operatorname{dim}(E \cap V) \leqq n-2 .
$$

The proof can be made by localizing the proof of the preceding theorem so it applies to a neighborhood of $x$. Of course use must be made of the fact that if $V$ is small enough, a compact subset $A$ of $V$ must separate $V$ if $H_{n-1}(A) \neq 0$. 


\section{BIBLIOGRAPHY}

1. Eilenberg and Steenrod, Foundations of algebraic topology, Princeton University Press, 1952.

2. Montgomery, Samelson, and Zippin, Singular points of a compact transformation group, Ann. of Math. vol. 63 (1956) pp. 1-9.

3. Montgomery, Samelson, and Yang, Exceptional orbits of highest dimension, Ann. of Math. vol. 64 (1956) pp. 131-141.

4. Gleason, Spaces with a compact Lie group of transformations, Proc. Amer. Math. Soc vol. 1 (1950) pp. 35-43.

5. Montgomery and Yang, Existence of a slice, Ann. of Math. vol. 65 (1957) pp. 108-116.

6. Mostow, Equivariant embeddings and euclidean spaces, Ann. of Math. vol. 65 (1957) pp. 432-446.

7. Smith, Transformations of finite period, II, Ann. of Math. vol. 40 (1939) pp. 690-711.

8. Yang, Transformation groups on a homological manifold, Trans. Amer. Math. Soc. vol. 87 (1958) pp. 261-283.

InSTITUTE For Advanced Study,

Princeton, N. J.

University of Pennsylvania,

Philadel phia, Pa. 